\title{
OTIOTOMICS
}

Revista de economía, empresa y sociedad

Dosier «Vectores de sostenibilidad: visiones desde la economía»

Coordinador: Albert Puig Gómez

\section{La financiarización del sector del agua}

\section{Hug March}

Universitat Oberta de Catalunya

RESUMEN Durante las últimas cuatro décadas, el centro del poder económico ha pasado de la industria a las finanzas. Como parte de esta tendencia, la «financiarización» del sector del agua ha añadido una nueva capa de complejidad al ciclo hidrosocial, testigo de la aparición de nuevos actores financieros, lógicas e instrumentos de financiación. Este cambio ha remodelado profundamente la relación entre las infraestructuras y empresas que intervienen en un bien esencial como el agua, la ciudadanía, los otros usuarios del agua y el medio ambiente. En este artículo, y basándome en trabajos previos, presento de manera resumida los debates en torno a la financiarización del ciclo hídrico, por medio del ejemplo de la provisión y del desarrollo de nuevas infraestructuras hídricas financiarizadas en la ciudad de Londres.

PALABRAS CLAVE economía política; financiarización; agua; privatización; Londres

\section{The financialization of the water sector}

ABSTRACT In the past four decades, the centre of economic power has moved from industry to finance. Against this backdrop, the financialization of the water industry has made the hydrosocial cycle more complex, witnessing the emergence of new financial logics and financing instruments. This change has thoroughly transformed the relation between infrastructures and water companies, citizens, other water users and the environment. In this article, and based on previous work, I present a summary that debates around the financialization of the water cycle, through the example of the provision and development of financialized water infrastructures in London.

KEYWORDS political economy; financialization; water; privatisation; London 


\section{Introducción}

En diciembre de 2020, surgió la noticia de que en California se había puesto en marcha un mercado de futuros del agua, pensado inicialmente para los agricultores, e intervenido por el Nasdaq Veles California Water Index (NQH2O), un índice creado que mide el precio al contado (spot price) de venta del agua en la zona. En estos mercados de futuros, como ya pasa en la energía o en otras materias primas, se "compra» la garantía de disponer del recurso en un momento concreto en el futuro. En otras palabras, no se compra el «recurso» físico en cuestión para uso inmediato en el momento de la transacción, sino que se «compra» la garantía de disponer del recurso en un momento concreto en el futuro. Si bien, desde un punto de vista ortodoxo, este tipo de mecanismos tendrían que llevar a una mejor utilización de los recursos y a cubrir de futuros riesgos de sequía a los agricultores u otros usuarios que participen en este mercado, en la práctica pueden llevar a una pura especulación de títulos de agua e incrementar las lógicas de extracción de beneficio privado de un bien esencial que es precio inelástico para los consumos más esenciales. Las ramificaciones y los impactos, todavía desconocidos, que la introducción de un mecanismo de compra de futuros sobre los derechos de agua puede tener son múltiples y afectan a diferentes actores y escalas espaciotemporales.

El ejemplo de California, a pesar de ser un caso extremo y minoritario, me sirve como punto de partida para iniciar una reflexión sobre el proceso de «financiarización» del sector del agua. Si bien los procesos de privatización han dominado las discusiones académicas sobre la gobernanza del agua desde finales de siglo, incluyendo los recientes debates en torno a la remunicipalización del agua, la «financiarización» del sector del agua o, en otras palabras, la introducción de nuevas lógicas e instrumentos financieros (como el caso de los mercados de futuro de agua o la indexación de productos financieros relacionados con el sector del agua como los Exchange Traded Funds) y la aparición de nuevos actores e intermediarios financieros (como fondos de inversión, fondo de pensiones, fondos soberanos, etc.) han añadido una nueva capa de complejidad al ciclo del agua. De hecho, las infraestructuras, sobre todo aquellas que intervienen en la circulación de recursos naturales vitales para la reproducción social y la actividad económica, como el agua o la energía, pero también las telecomunicaciones o las vías de transporte, entre otros, se han convertido en objetos cruciales para la acumulación de capital en la economía global en las últimas décadas, tal como argumenta Torrance (2009). En paralelo, las lógicas de financiarización del agua mimetizan otros procesos de financiarización del medio ambiente (Loftus y March, 2015).

Como dice el geógrafo Brett Christophers (2015), si la globalización fue el concepto clave utilizado (y sobreutilizado) tanto por académicos como activistas para dar sentido (y criticar) las reconfiguraciones de la economía en la década de 1990 y la noción de neoliberalización lo fue ya en el siglo XXI, el concepto de financiarización sería uno de los neologismos más utilizados para dar sentido a las reconfiguraciones político-económicas que redibujan nuestra sociedad desde hace una década. Pero ¿qué entendemos por financiarización? Ben Fine (2013) argumenta que la financiarización se define por la importancia creciente de aquello que se conoce como interest bearing capital (capital con interés; es decir, dinero que se presta para ganar más dinero). La dependencia creciente de la rentabilidad futura (de carácter especulativo) asociada con la circulación creciente de este capital con interés conduce a una expansión de lo que David Harvey (2006) denomina capital ficticio: en otras palabras, dinero que se lanza a la circulación como capital sin ninguna base material/biofísica o actividad productiva asociada.

Este trabajo, que recoge aportaciones hechas en investigaciones previas del autor con otros coautores, traza y caracteriza algunos de estos procesos de financiarización del agua, y de sus infraestructuras, por medio de ejemplos en diferentes temporalidades y espacialidades, especialmente el Reino Unido y, más concretamente, el caso de Thames Water, proveedor de agua a unos 15 millones de personas del área metropolitana de Londres y su región de influencia. 


\section{De la privatización a la financiarización: un breve recorrido por la economía política del ciclo del agua inglés}

El caso de la financiarización de la infraestructura del agua en el Reino Unido y, más concretamente, en Londres, ha recibido la atención de diferentes académicos (entre otros, Allen y Pryke, 2013; Bayliss, 2017; Pryke y Allen, 2019) dado su carácter extremo, que sirve de antesala para entender cómo la financiarización puede reconfigurar el sector del agua de otras geografías. De hecho, el proceso de «privatización total» (full divesture) del ciclo del agua en Inglaterra y Gales ha sido estudiado ampliamente desde diferentes campos, incluyendo aproximaciones geográficas y de ecología política. En 1989, la entonces primera ministra Margaret Thatcher emprendió la privatización (full divesture) de las compañías públicas regionales que gestionaban el suministro y saneamiento del agua a escala regional/de cuenca hidrográfica en Inglaterra y Gales. La privatización de Thames Water se enmarca en este proceso de reconfiguración radical de la naturaleza del ciclo integral del agua inglés y galés. Durante los primeros cinco años después de la privatización, el Gobierno del Reino Unido tuvo una "acción dorada» en estas compañías, al evitar cualquier cambio dramático en la propiedad y asegurar que Thames Water se adhiriera al tipo de modelo de «capitalismo popular» thatcheriano. Aun así, después de unos años iniciales en los que el capital (privado) de las compañías era mayoritariamente británico, a partir de la segunda mitad de la década de 1990 hubo una entrada masiva de capital internacional, mayoritariamente de compañías de agua que dominaban el mercado mundial en aquella época. De este modo, en 2001, Thames Water fue comprada por la compañía alemana multiservicios RWE.

Un segundo cambio importante en la economía política del agua en Inglaterra tuvo lugar en la segunda mitad de la primera década del siglo XXI, con la entrada masiva de nuevos actores en las estructuras de propiedad de las compañías privadas de agua, lo que sustituyó totalmente en muchos casos el capital industrial internacional. De hecho, la entrada de nuevos actores y lógicas financieras empezó a reconfigurar el mercado del agua global una vez en el siglo XXI (March y Purcell, 2014), no solo en el Reino Unido, sino directamente en otros países, como Chile, país que había vivido una privatización muy intensa del ciclo del agua. Volviendo a Inglaterra, en 2006, la propiedad de Thames Water pasó a manos de Kemble Water Holdings Ltd., un conglomerado de inversores, liderado en aquel momento inicial por el fondo australiano de inversiones Macquarie, con una amplia cartera de infraestructuras.

En septiembre de 2021, ${ }^{1}$ entre los accionistas principales de este complejo entramado financiero se encuentra Ontario Municipal Employees Retirement System (31,7 \%), uno de los fondos de pensiones más grandes de Canadá, seguido del fondo de pensiones de los profesores universitarios del Reino Unido, Universities Superannuation Scheme (alrededor de un $11 \%$ ). Con participaciones inferiores al $10 \%$, hay un fondo subsidiario del Abu Dhabi Investment Authority, uno de los fondos soberanos más grandes del mundo; Wren House, un vehículo de inversiones en infraestructuras de la Kuwait Investment Authority, otro de los fondos soberanos más grandes del mundo; British Columbia Investment Management Corporation, un servicio de gestión de inversiones que provee al sector público de la Columbia Británica, en Canadá; Hermes GPE, uno de los gestores líderes de inversiones en mercados globales de capital; China Investment Corporation, uno de los fondos soberanos más grandes del mundo; Queensland Investment Corporation, uno de los gestores de fondos institucionales más grande de Australia; el fondo de infraestructuras Aquila GP Inc.; y el Stichting Pensioenfonds Zorg en Welzijn, el segundo fondo de pensión más grande de los Países Bajos. Vemos que Thames Water refleja a la perfección lo que se puede entender por financiarización de compañías/infraestructuras de servicios básicos para la vida, como el agua: presencia de fondo de pensiones (públicos, en muchos casos) de Canadá, del Reino Unido o de los Países Bajos, fondos soberanos de países de Oriente Próximo y de China, y también presencia de vehículos financieros australianos.

Estas nuevas coreografías en las estructuras de propiedad de las compañías de agua inglesas, que destacan en el caso concreto de Thames Water, tienen un impacto y condicionan de manera directa el desarrollo de nuevas infraestructuras hídricas que, de paso, posibilitan un sistema de extracción de rentas que amplifica las lógicas establecidas en los procesos de privatización. Pryke y Allen (2017), que ya habían iniciado el trabajo crucial sobre financiarización del agua en el Reino Unido, ilustran varios de estos cambios en la provisión de infraestructuras financiarizadas con el caso de la desalinizadora de Carlsbad, en San Diego, California. Mediante un análisis particularmente detallado del

1. https://www.thameswater.co.uk/about-us/governance/our-structure 
abanico de actores financieros y paquetes de inversión que posibilitaron el desarrollo de esta infraestructura, los autores demuestran la capacidad de los intermediarios financieros de extraer valor de los activos fijos y convertirlos en activos líquidos. La titulización de activos, como ya se ha descrito anteriormente, es una parte crucial del proceso que estos autores describen: por medio del empaquetamiento de los flujos de ingresos garantizados (presentes y futuros) como mercancía financiera, una serie de inversores son capaces de «extraer valor» de esta infraestructura. En este sentido, Alhers y Merme (2016) hablan de un cambio radical en el modelo de financiación de las infraestructuras hídricas que, de este modo, condiciona su provisión y desarrollo.

Desarrollando este hilo argumental en torno a las infraestructuras financiarizadas, y siguiendo con el caso de Londres, en el artículo «Financializing desalination: Rethinking the returns of big infrastructure» (Loftus y March, 2016) documentamos el desarrollo de la desalinizadora más grande del Reino Unido, en la cuenca del Támesis, operada por Thames Water. En este artículo reflexionamos sobre cómo el modelo financiarizado de la compañía, y sus dinámicas de apalancamiento financiero y búsqueda interminable de nuevas oportunidades de negocio, habían condicionado la existencia y el desarrollo de esta infraestructura, con un coste de 270 millones de libras esterlinas. Años después de la elaboración de este artículo, vimos cómo el orden de magnitud de esta inversión no era nada comparado con los billones de libras destinadas a uno de los megaproyectos del siglo XXI en la ciudad de Londres: el Thames Tideway Tunnel, un megatúnel destinado a mejorar la gestión de aguas residuales en la ciudad, cuya construcción se inició en 2018 y debería finalizar en 2023, con un coste estimado por encima de los 4.000 millones de libras esterlinas. Siguiendo nuestros argumentos en torno a los impactos de la financiarización en la forma, el tamaño y los objetivos finales de la infraestructura hídrica, en el artículo «Integrating what and for whom? Financialisation and the Thames Tideway Tunnel» (Loftus y March, 2019) nos centramos en entender la relación entre esta gran infraestructura y la intensa financiarización de la compañía de agua londinense, y documentamos el complejo entramado financiero para atraer el capital necesario para desarrollarla y las ramificaciones que esto ya tenía en los hogares londinenses: unos cuantos años antes de la apertura del túnel, las facturas anuales de los hogares ya habían aumentado de media en 18 libras para financiar el túnel, con estimaciones de aumento medio anual de cerca de 20-25 libras si el regulador público lo permite (Financial Times, 11 de julio de 2021). ${ }^{2}$ De hecho, el argumento de cómo la financiarización del agua en el Reino Unido impactaba de manera material en los hogares ya lo había empezado a desarrollar Allen y Pryke (2013), y también había sido desarrollado por Loftus et al. (2016), con la demostración empírica de cómo «el hogar» se había convertido en un activo líquido para las empresas de servicios básicos financiarizados.

\section{Conclusión}

A comienzos del siglo XXI, los debates académicos sobre la economía política del agua giraban alrededor de la transformación de las aguas «locales» en flujos monetarios "globales» e ilustraban la reconfiguración radical del sector del agua por medio de los procesos de privatización del suministro en múltiples geografías del norte y del sur global. En la tercera década del siglo XXI, y sin menospreciar la relevancia que continúan teniendo los procesos de privatización (y también de remunicipalización) en las realidades materiales de millones de personas, los tiempos actuales requieren asimismo centrarnos en la financiarización, un proceso de mutación e intensificación de las lógicas de acumulación y de reproducción del capital privado en la provisión de un recurso esencial.

Como hemos visto en el caso inglés, que hemos expuesto en este artículo, pero también como se puede observar en procesos de financiarización del ciclo del agua en otras comarcas, como California, los flujos de ingresos que emanan de los activos de infraestructura y de los derechos de propiedad del agua se transforman en materias primeras «líquidas» financieras que garantizan la extracción continuada de rentas del ciclo hidrosocial por Estados (por medio de fondos soberanos), pensionistas (por medio de fondos de pensiones) y otros tipos de inversores (por medio de fondos de inversión y otros vehículos financieros) de todo el mundo. A pesar de que esto no siempre puede ser un movimiento negativo - de hecho, para Noel Castree y Brett Christophers (2015), la entrada de nuevos recursos financieros puede proporcionar un recurso crucial para las transformaciones sostenibles-, en muchos casos, la financiarización favorece un proceso de acumulación por desposesión en el que elementos centrales en nuestra reproducción social, como los

2. https://www.ft.com/content/f25e29f9-03b4-43a2-9da5-779bcdc3f883 
recursos naturales, dependen de un modelo financiero cada vez más arriesgado y fundamentalmente no democrático que privilegia los beneficios de los inversores por encima de las necesidades básicas de la ciudadanía y de la realidad biofísica de estos recursos naturales. En un contexto global en el que una parte nada despreciable de la población tiene dificultades para tener un acceso de calidad al agua potable y al saneamiento, situación que se intensifica con la emergencia climática, hay que recuperar mecanismos de gobernanza democrática, inclusiva y equitativa del agua. Se pone en entredicho que los procesos de financiarización discutidos en este artículo precisamente vayan en esta dirección.

\section{Referencias bibliográficas}

AHLERS, R.; MERME, V. (2016). «Financialization, water governance, and uneven development». En: Wires Water, vol. 3, núm. 6, págs. 766-774 [en línea]. DOl: https://doi.org/10.1002/wat2.1166

ALLEN, J.; PRYKE, M. (2013). «Financialising household water: Thames Water, MEIF, and 'ring-fenced'politics». En: Cambridge Journal of Regions, Economy and Society, vol. 6, núm. 3, págs. 419-439 [en línea]. DOl: https://doi. org/10.1093/cjres/rst010

BAYLISS, K. (2017). «Material cultures of water financialisation in England and Wales». En: New Political Economy, vol. 22, núm. 4, págs. 383-397 [en línea]. DOI: https://doi.org/10.1080/13563467.2017.1259300

CASTREE, N.;CHRISTOPHERS, B. (2015). «Banking Spatially on the Future: Capital Switching, Infrastructure, and the Ecological Fix». En: Annals of the Association of American Geographers, vol. 105, núm. 2, págs. 378-386. DOl: https://doi.org/10.1080/00045608.2014.985622

CHRISTOPHERS, B. (2015). «Geographies of finance II: Crisis, space, and political-economic transformation». En: Progress in Human Geography, vol. 39, págs. 205-213 [en línea]. DOI: https://doi.org/10.1177/0309132513514343

FINE, B. (2013). «Financialization from a Marxist perspective». En: International Journal of Political Economy, vol. 42, núm. 4, págs. 47-66 [en línea]. DOI: https://doi.org/10.2753/IJP0891-1916420403

HARVEY, D. (2006 [1982]). The Limits to Capital. Londres: Verso.

LOFTUS, A.; MARCH, H (2015). «Financialising nature?». En: Geoforum, vol. 60, págs. 172-175 [en línea]. DOI: https:// doi.org/10.1016/j.geoforum.2015.02.004

LOFTUS, A.; MARCH, H. (2016). «Financializing desalination: Rethinking the returns of big infrastructure». En: International Journal of Urban and Regional Research, vol. 40, núm. 1, págs. 46-61 [en línea]. DOl: https://doi.org/10.1111/14682427.12342

LOFTUS, A.; MARCH, H.; NASH, F. (2016). «Water Infrastructure and the Making of Financial Subjects in the South East of England». En: Water Alternatives, vol. 9, núm. 2.

LOFTUS, A.; MARCH, H. (2019). «Integrating what and for whom? Financialisation and the Thames Tideway Tunnel». En: Urban Studies, vol. 56, núm. 11, págs. 2280-2296 [en línea]. DOI: https://doi.org/10.1177/0042098017736713

LOFTUS, A.; MARCH, H.; PURCELL, T. F. (2019). «The political economy of water infrastructure: An introduction to financialization». En: Wiley Interdisciplinary Reviews: Water, vol. 6, núm. 1, e1326 [en línea]. DOl: https://doi. org/10.1002/wat2.1326

MARCH, H.; PURCELL, T. (2014). «The muddy waters of financialisation and new accumulation strategies in the global water industry: The case of AGBAR». En: Geoforum, vol. 53, págs. 11-20 [en línea]. DOI: https://doi.org/10.1016/j. geoforum.2014.01.011

PRYKE, M.; ALLEN, J. (2017). «Financialising urban water infrastructure: Extracting local value, distributing value globally». En: Urban Studies, vol. 56, núm. 7, págs. 1326-1346 [en línea]. DOl: https://doi.org/10.1177/0042098017742288

TORRANCE, M. (2009). «The rise of a global infrastructure market through relational investing». En: Economic Geography, vol. 85, núm. 1, págs. 75-97 [en línea]. DOl: https://doi.org/10.1111/j.1944-8287.2008.01004.x 
Cita recomendada: MARCH, Hug. La financiarización del sector del agua. Oikonomics [en línea]. Noviembre 2021, n. 16. ISSN: 2339-9546.

DOI: https://doi.org/10.7238/o.n16.2112

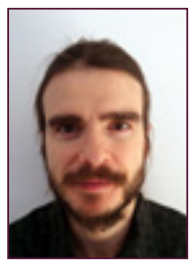

\section{Hug March}

hmarch@uoc.edu

Universitat Oberta de Catalunya

Profesor de los Estudios de Economía y Empresa de la Universitat Oberta de Catalunya (UOC). Imparte docencia de asignaturas relacionadas con la sostenibilidad en diferentes grados y másteres. Es también investigador en el Laboratorio de Transformación Urbana y Cambio Global (TURBA) del Internet Interdisciplinary Institute, UOC. Su investigación gira alrededor de la ecología política, desde la gestión del agua hasta cuestiones más amplias de sostenibilidad, con un foco especial en la dimensión urbana y metropolitana.

Los textos publicados en esta revista están sujetos -si no se indica lo contrario- a una licencia de Reconocimiento 4.0 Internacional de Creative Commons. Puede copiarlos, distribuirlos, comunicarlos públicamente, hacer obras derivadas siempre que reconozca los créditos de las obras (autoría, nombre de la revista, institución editora) de la manera especificada por los autores o por la revista. La licencia completa se puede consultar en https://creativecommons.org/licenses/by/4.0/deed.es_ES.

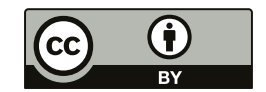

